\title{
The benefits of individual versus group work in a biology based laboratory setting
}

Cronin, Michael

University College Cork, Ireland (m.cronin@ucc.ie)

McCabe, Anthony $\bowtie$

Institute of Technology Sligo, Ireland (mccabe.tony@itsligo.ie)

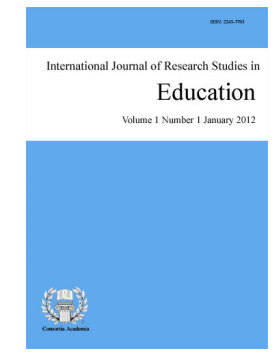

ISSN: 2243-7703 Online ISSN: 2243-7711

OPEN ACCESS

\section{Abstract}

Laboratory science classes and group work are almost inextricably linked in student college life today. The merits of both are extolled by both educators and future employers as a fundamental part of a rounded qualified graduate. However, there is little evidence to the contrary, particularly in laboratory based programs. This study aims to determine the students' perception of the benefits of different working scenarios in a biological laboratory setting. The study followed 32 students in their third year of their college program working in three different biology based laboratory classes. During each of these laboratory classes the students either worked alone, worked with a preferred partner or worked with an assigned partner. The students were asked to grade their experiences in a survey. The students were asked to reflect on the following areas: enjoyment of experience, understanding of activities, laboratory technique and time efficiency. Mixed modal analysis of variance and pair-wise analyses were used to determine significance between the differing work group scenarios. The laboratory group make-up had no impact on the student enjoyment of the laboratory class. There was also no significant impact on the students' ability to perform the laboratory exercise in a time efficient manner. The analysis did however indicate that students who work alone or with their preferred partner have a greater understanding of the subject matter against those who worked with an assigned partner. The results also suggest that students who worked alone had significantly improved laboratory technique compared to student working in a group.

Keywords: working alone; technique; teamwork; individual 


\section{The benefits of individual versus group work in a biology based laboratory setting}

\section{Introduction}

Modern curriculum and teaching practices incorporate team work as part of their teaching and assessment design. The reasons for this emanate from academic studies which seem to suggest benefits from working in groups. Effective team work is viewed as crucial in getting students work ready. In a corporate setting, work teams are almost universal and are formed in design, manufacturing, technical and sales divisions for product research, development and marketing.

The experience of being in a functioning, successful team can be hugely beneficial to a student's skill set and include:

Outcomes are often of a higher standard.

$>$ Peer and co-operative learning.

Active participation and confidence in part due to group supports.

$>$ A wider knowledge base through pooling of information and research skills, or peer-tutoring can lead to a diverse knowledge base for team members.

$>$ Discussion, debate, explanation, argument, justification of views within a team setting promotes understanding.

$>$ The acceptance of transferable skills such as time management, written communication and leadership skills.

> Enhanced motivation through the formation of friendships (Dunne \& Rawlins, 2000; Davies, 2009; Millis, 2010; De Grave, Boshuizen, \& Schmidt, 1996).

Despite this, some students do not function well or don't experience the benefits as outlined above. Common difficulties and concerns experienced by students working in groups include:

$>$ Not all students learn everything about the topic, especially if the task and workloads are divided into separate components.

$>$ Some students prefer to work and be assessed independently.

$>$ There is an uncertainty factor in group work compared to individual assignments.

$>$ Students can feel as if they have been 'thrown in at the deep end' if they don't have adequate support.

$>$ Some students in a group don't contribute or commit equally.

$>$ Individual grades may be affected if an overall group mark is awarded.

$>$ Some people tend to dominate others in the team or the team activities.

$>$ Internal team dynamics can collapse.

> 'Groupthink' can take over where all members follow a leader and effectively (unconsciously or not) come to the same conclusion as everyone else, individual analysis and ideas can be diminished (Watson, 2002; Levin, 2005; Davies, 2009).

University and college science laboratory classes are conventionally conducted with students in groups of two or more. While evidence in the literature to support this assertion is lacking, it is certainly the experience of many (personal communication). The likely reasons for this emanate (at least initially) from the advantages which come with working within a team. However, class management is also a pertinent reason for running classes in groups; less actual laboratory space is required for classes run in groups meaning fewer classes need to be schedules. Fewer work stations also makes managing a laboratory full of students more efficient. Inevitably, group work also results in reduced costs (including technical time spent in preparation) compared to setting up individual work stations for students. In an increasingly competitive education sector, prudent financial management has become a considerable issue.

Given this approach to laboratory teaching, the question arises as to whether we are sacrificing student learning outcomes through running laboratory classes in groups as opposed to allowing students to work on their own. What are the perceived benefits from the point of view of the students? 
The benefits of individual versus group work in a biology based laboratory setting

Dunne and Rawlins (2000) have suggested that one of the key advantages of team work is that friendship and membership of a community can be strengthened, with evidence of enhanced motivation as a consequence. Students who are friends may work well together because there is a confidence and trust between them and they are mindful of each other's strengths and weaknesses. However, contrary to this, friends which form groups may also be detriment to the success and objectives of the group. Oakley and associates (2003) suggest that close friends working together on projects are inclined to 'circle the wagons and look inwards', protecting each other and participating in plagiarism. Our own experience of self-formed groups between friends is that they can easily distract each other with social commentary, social media devices and social networking rather than partaking in group activities. Encountering students on networking sites during laboratory classes is not an uncommon occurrence and is becoming increasingly problematic (McCoy, 2013). Some students are likely to view groups formed between friends as more of an opportunity to play and socialize rather than work (McDonald, 2016; Cohen \& Lotan, 2014).

There is an argument that working alone may in fact be a superior approach, at least in some respects. Lone working would certainly overcome issues outlined by Levin (2005) and Watson (2002), such as team dynamics collapsing or one team member dominating over the others. It also means that the student working on their own is involved in every facet of the project in question rather than being allocated part of the project within a team scenario and thus helping to develop a range of skillsets. Students working on their own cannot rely on others and must focus to get projects completed as there is no shared responsibility. They are also less likely to be diverted by the trappings of self-formed groups such as social distractions. Significantly, Welsh et al. (2007) demonstrated that students working together may actually impede each other. In a student partnership on a computer laboratory task, one partner watching the other perform a task caused the student to perform the task slower and with less accuracy. Welsh et al. suggest that certain tasks which require speed and accuracy may be better taught in an individual scenario rather than in a group environment.

In this current study, students from a Biotechnology program were exposed to different working group scenarios over three separate laboratory modules in a single academic year. The students either worked on their own, worked within an assigned group of two or worked in a 'self-formed' group of two. At the end of the academic year all students were given a short questionnaire on their experiences. Students were questioned on their preferences for each work setting and how they felt the differing work settings effected their enjoyment, understanding, their technique and their time management.

\section{Methods}

\subsection{Class background}

The participants in the study consisted of 32 students, from the Institute's Medical Biotechnology and Biomedical Sciences programmes. Both these programmes study a number of modules in common and the study was performed in three laboratory based modules; animal cell culture, implant biocompatibility and immunodiagnostics. All three modules are taught at level 7 (nfq.ie, 2016). All three modules are taught by the same lecturer.

\subsection{Study design}

At the beginning of the academic year the students asked to notify the module organizers by email if they had a preferred laboratory partner. It was explained to the students that during the academic year they would be placed in laboratory groups with their preferred partner (if they supplied one), with a partner assigned by the lecturer or they would work on their own. Of the 32 students, only 12 listed a preferred partner and so only these 12 could be studied for comparison between preferred partner, assigned partner and working alone. Three different laboratory based modules were used in the study. One of the modules, animal cell culture, had a total laboratory time of 30 hours over 11 weeks in semester one. The other modules involved in the study, implant 
biocompatibility and immunodiagnostics each contained 24 hours of laboratory work over a 4-week period in semester 2. The timing of the Implant Biocompatibility and Immunodiagnostics modules did not overlap during semester 2.

\subsection{Student questionnaire}

At the end of the academic year all students involved in the study (including those who did not indicate a preferred laboratory partner) were asked to complete a questionnaire of their experiences. The questionnaire is outlined in Figure 1. The questionnaire was handed out during class at the end of the academic year and was completed anonymously.

\subsection{Statistical Analysis}

Questionnaire and laboratory marks were summarised using means and standard deviations. Questionnaire answers and laboratory marks were compared between groups/treatments (Alone/Preferred/Assigned) using mixed models analysis of variance. The group/treatment was a fixed effect. An appropriate variance-covariance structure was selected using Akaike information criteria to account for the expected correlation between responses from individual students. A variance components structure was selected for Question 3 (Enjoyment), unstructured matrices were selected for the other 3 questions (understanding, technique and efficiency) and a compound symmetry structure was selected from laboratory marks. The GPA was included as a covariate for the analysis of laboratory marks, thus adjusting the comparisons between groups/treatments for any effect for GPA. When a significant difference was detected, pair-wise comparisons were performed, adjusted using the Sidak method. Statistical analyses were performed in SAS (Version 9.4).

\section{Q1. Which (type of work scenario) did you prefer? \\ Working on your own \\ Working with your preferred partner (mark N/A if not applicable) \\ Working with your assigned partner}

\section{Q 2. Would you like more practical classes working on your own? (Circle answer) \\ Yes No No preference}

Q3. On a scale of 1-5 how much did you enjoy working on your own/with your assigned partner/with your preferred partner? 5 being the maximum score.
1(lowest score)
4
5 (highest score)

Did it help your understanding of the practical experiment?

$\begin{array}{lllll}\text { 1(lowest score) } & 2 & 3 & 4 & 5 \text { (highest score) }\end{array}$

Did it help your laboratory technique?

$\begin{array}{lllll}1 \text { (no help) } & 2 & 3 & 4 & 5 \text { (most helpful) }\end{array}$

Did it make you more efficient, timewise? (Did you find you completed the laboratory class ahead of time?)

$\begin{array}{lllll}\text { (less efficient) } & 2 & 3 & 4 & 5 \text { (most efficient) }\end{array}$

Figure 1. Student questionnaire was circulated to all 32 students who had attended the three laboratory classes. Question 3 was asked for each of the types of teams (work alone, work with preferred partner, work with assigned partner).

\section{Results}

\subsection{Student Data Set}

At the beginning of the academic year, the class of 32 students were emailed and requested that the submit the name of their preferred working partner for laboratory classes. 12 matched preferred-partner groups were 
The benefits of individual versus group work in a biology based laboratory setting

formed in responses received from students. There were no scenarios whereby a student listed another student as a preferred partner who did not respond in kind. Whichever working group scenario (alone, assigned, preferred) the student was placed in at the beginning of a set of laboratory classes, they remained in that scenario for the entire length of that laboratory class.

Of the 32 students, 12 of them ultimately ended the academic year having experienced all three treatments; working alone, working with an assigned partner or working with a preferred partner. In some cases, students may have worked alone or with an assigned partner or preferred partner on more than one occasion. This discrepancy is in part due to laboratory space issues limiting the number of students working alone or in some circumstances whereby reagent preparation and availability meant that some classes had to run with a higher number of groups. Class management issues such as students being unavailable during laboratory sessions also meant that some students were unavailable to fit into group scenarios when needed.

\subsection{Student Preferences}

The first question put to the students at the end of the academic year was which work scenario they preferred most; working alone, working with their preferred partner or working with an assigned partner. The results are illustrated in figure 2. 21 student responses are included in the data presented in figure 2. Two student's responses were omitted for these questions as more than one answer for question 1 was ticked. Figure 2 illustrates preference for either working alone or with any partner (either assigned or preferred). Figure 2 shows that $76.2 \%$ of students indicate a preference for working alone against $23.8 \%$ who prefer to work with a partner.

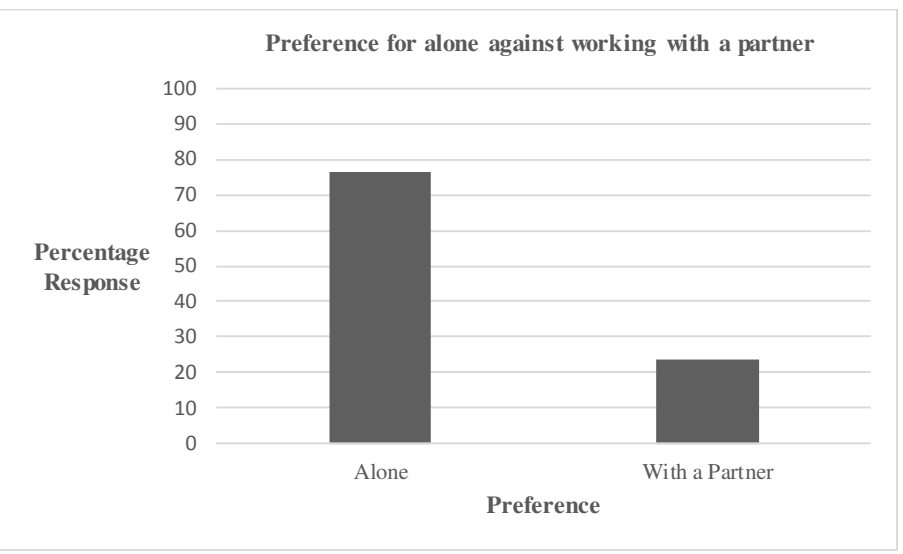

Figure 2. Illustrates students answers to question 1 of survey. Figure 2 does not discriminate between the type of group which the students worked in and illustrates whether the students preferred working alone or within any type of grouping (preferred or assigned), $n=21$.

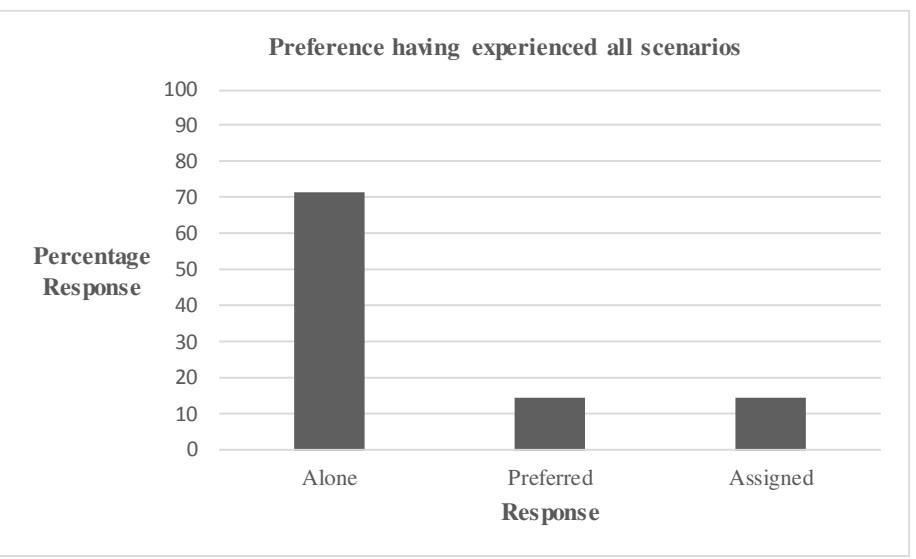

Figure 3. Illustrates students answers to question 1 of survey but excludes those students for whom the survey indicated they had not experienced all three work scenarios, $n=14$. 
Figure 3 shows the results for question one for those students who experienced all three work scenarios, when those students who indicated they did not have either a preferred or assigned partner are omitted. The results illustrated in figure 2 are representative of 14 student responses, 2 more than the 12 students who were assigned all three scenarios during the academic year. We can only assume that one student answered question 1 based on his/her past experiences of working alone or with an assigned or preferred partner. Of those who experienced all three work scenarios, $71.4 \%$ indicate they preferred working alone against $14.3 \%$ who preferred working along their preferred partner and an equal number, $14.3 \%$, indicated they preferred working alongside an assigned partner.

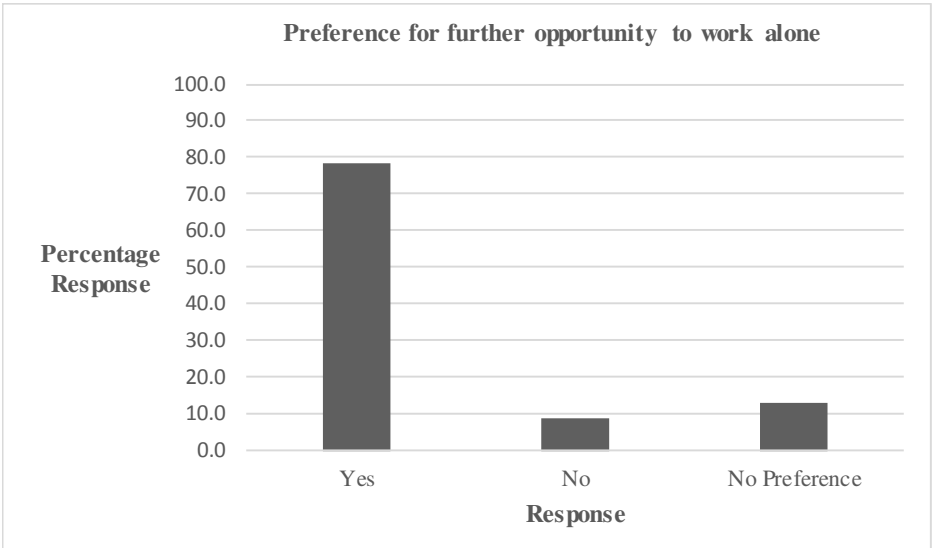

Figure 4. Students were asked if they would like the opportunity to work alone in laboratory classes more often. $78.3 \%$ students answered yes, $8.7 \%$ answered no and $13 \%$ of students had no preference. $\mathrm{N}=23$.

Question 2 asked the students if they would like the opportunity to work alone again in laboratory classes. Once again, an overwhelming majority, $78.3 \%$, indicated they would like that opportunity against $8.7 \%$ who said no and $13 \%$ who had no preference, see figure 4 . On occasion, given the hours and sometimes the cost involved in running cell culture or antibody based laboratory classes (such as immunodiagnostics), laboratory groups can contain more than two students. Though this was not in the case in this current study, students either worked alone or in a group of 2, students were asked their opinion on group sizes in laboratory class. $100 \%$ of students indicated that laboratory groups should contain no more than two students (data not shown).

\subsection{Effects of groups on Enjoyment, Understanding, Laboratory Technique and Time Efficiency}

Students were asked to rate the effect of the working group scenario on their enjoyment of the laboratory, their understanding of the laboratory activities, whether it helped their technique and did it effect how efficient they were in the laboratory. Students scaled these answers from 1-5 as outlined in the questionnaire in figure 1. All students were included in this analysis regardless of whether they experienced all three group scenarios. The questionnaire indicated that 23 students worked alone during at least one laboratory class, 17 students worked with a preferred partner in at least one laboratory class and 22 students worked with an assigned partner during at least one laboratory class.

Student Enjoyment - The initial question asked of students was to determine was there a grouping which they enjoyed more than another. Effects were compared between groups/treatments (Alone/Preferred/Assigned) using mixed models analysis of variance. Analysis indicates no significance difference between treatment groups and are illustrated in Table 1.

Student Understanding -Table 2 illustrates analysis of student responses (on a scale of 1-5) on the impact of group type on their understanding of the experiment. Effects were compared between groups/treatments (Alone/Preferred/Assigned) using mixed models analysis of variance. ANOVA analysis demonstrated a significant difference between within the treatments, $p=0.0098$, see Table 2. Subsequent pair-wise analysis 
The benefits of individual versus group work in a biology based laboratory setting

showed that understanding of the experiment was significantly enhanced when working alone against working with an assigned partner $(p=0.0464)$ and when working with a preferred partner as opposed to working with an assigned partner $(p=0.0464)$.

Table 1

Impact of student working group on enjoyment

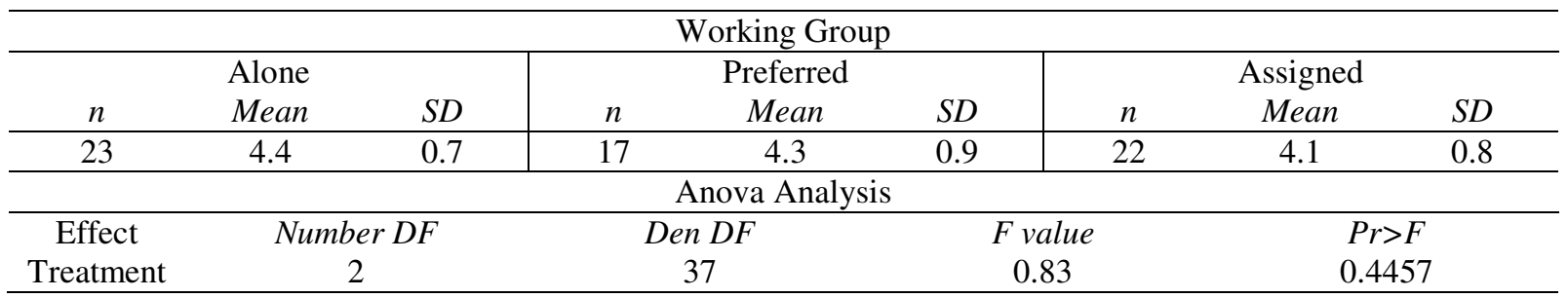

Results are based on a scale of 1-5 (5 indicating most enjoyment and 1 indicating least).

Table 2

The impact of student working group on understanding

\begin{tabular}{|c|c|c|c|c|c|c|}
\hline \multicolumn{7}{|c|}{ Working Group } \\
\hline \multicolumn{3}{|c|}{ Alone } & \multicolumn{2}{|c|}{ Preferred } & \multicolumn{2}{|c|}{ Assigned } \\
\hline$n$ & Mean & $S D$ & Mean & $S D$ & Mean & $S D$ \\
\hline 23 & 4.3 & 0.7 & 4.3 & 1 & 3.6 & 1.1 \\
\hline \multicolumn{7}{|c|}{ Anova Analysis } \\
\hline Effect & \multicolumn{2}{|c|}{ Number DF } & Den $D F$ & \multicolumn{2}{|c|}{ F value } & \\
\hline Treatment & \multicolumn{2}{|l|}{2} & 22 & 5.75 & & \\
\hline \multicolumn{7}{|c|}{ Note. Differences statistically significant $(p=0.0098)$. } \\
\hline \multicolumn{7}{|c|}{ Pair-wise comparisons (adjusted) } \\
\hline Effect & Treatment & & \multicolumn{4}{|l|}{ Adjusted P } \\
\hline Treatment & Alone & $\begin{array}{l}\text { 1 reatment } \\
\text { Assigned }\end{array}$ & 0.0464 & & & \\
\hline Treatment & Alone & Preferred & 1 & & & \\
\hline Treatment & Assigned & Preferred & 0.048 & & & \\
\hline \multicolumn{7}{|c|}{$\begin{array}{l}\text { Note. Working alone has higher score than working with an assigned partner }(p=0.0464) \text {. } \\
\text { Working with a preferred partner has higher score than with an assigned partner }(p=0.048) \text {. }\end{array}$} \\
\hline \multicolumn{7}{|c|}{$\begin{array}{l}\text { Laboratory Technique - Table } 3 \text { addresses the questions of laboratory technique. When asked how each } \\
\text { tment affected their laboratory technique there was noticeable and significant differences between the } \\
\text { upings. Effects were compared between groups/treatments (Alone/Preferred/Assigned) using mixed models } \\
\text { lysis of variance, which indicated significant differences. Pair-wise analysis of the student response indicates } \\
\text { laboratory technique was significantly improved in students who worked alone against those working with } \\
\text { er a preferred partner or an assigned partner, see Table } 3 \text {. }\end{array}$} \\
\hline
\end{tabular}

Table 3

The Impact of student working group on laboratory technique

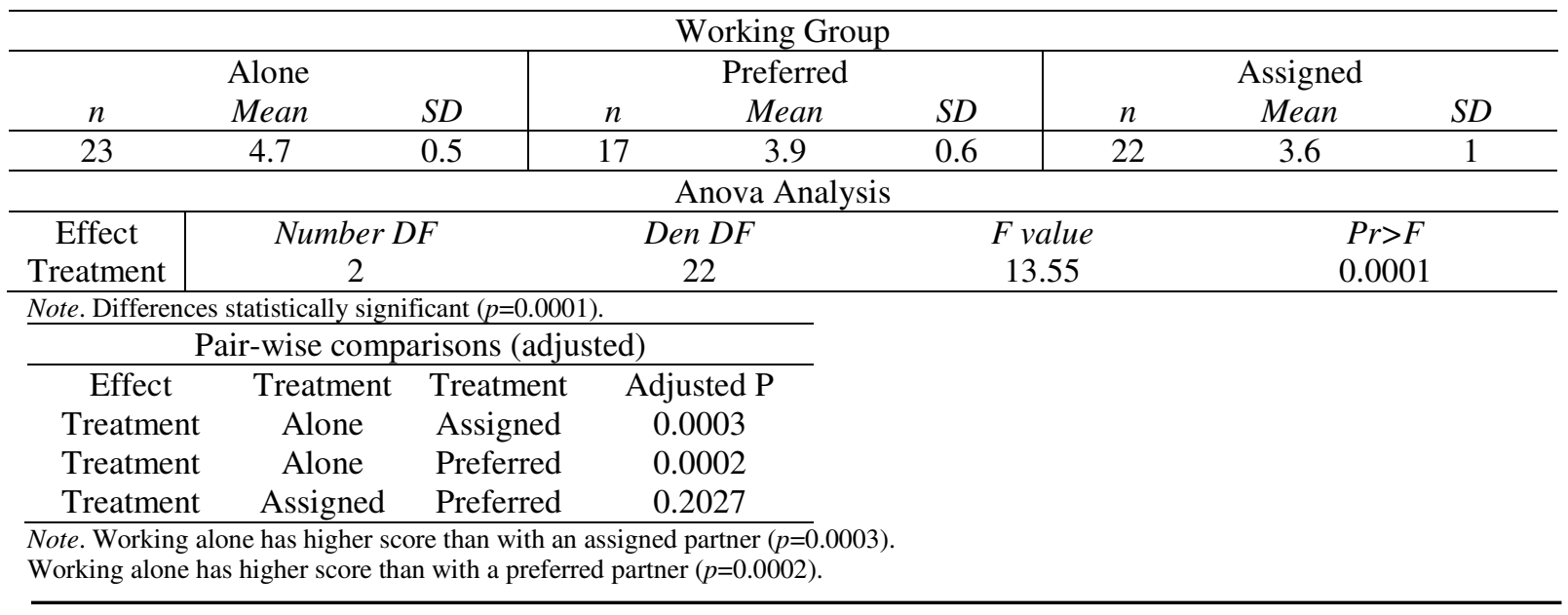


Efficiency - Students were asked how efficiency was impacted by the three treatments. Efficiency in this case was defined by the time taken to complete the laboratory class. Once again, students were asked on a scale of 1-5 how efficiently they performed the experiments in under the different groupings. Effects were compared between groups/treatments (Alone/Preferred/Assigned) using mixed models analysis of variance. Results are shown in Table 4 and indicate no differences between the treatment groups.

Table 4

The effect of student grouping on time taken to complete experiments

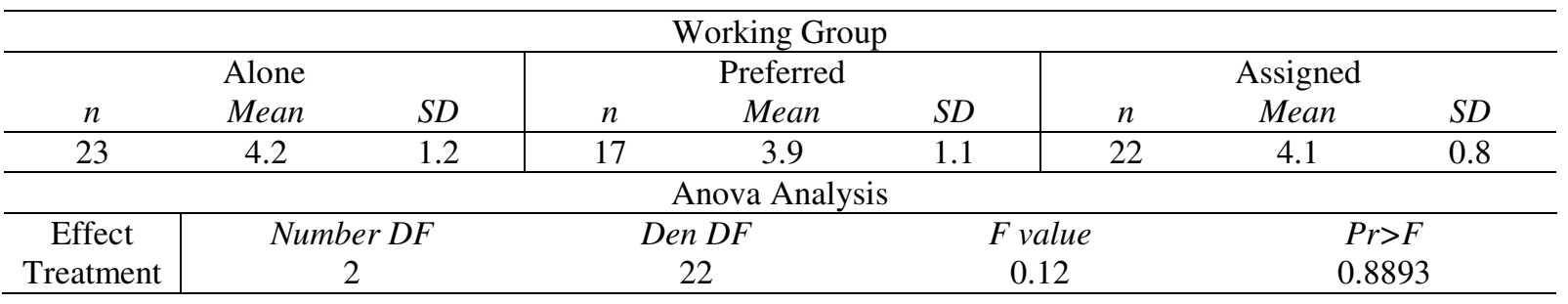

Table 5

The effect of student work group on overall laboratory score

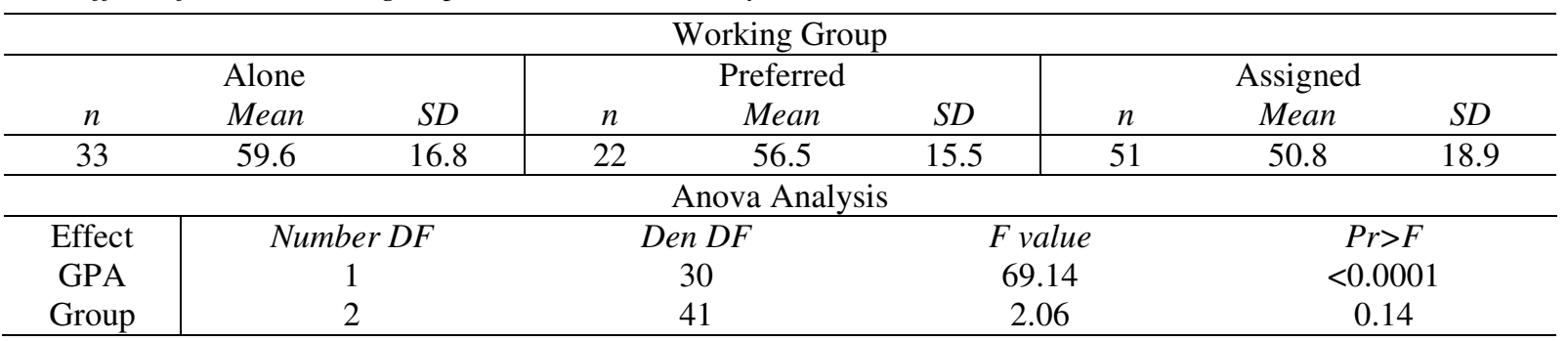

Note. Differences not significant $(p=0.14)$. GPA $=$ Grade Point Average.

Analysis of laboratory mark against groups/treatments (Alone/Preferred/Assigned) using mixed models analysis of variance

\section{Discussion}

The importance of student group work in academic settings cannot be understated. Team assignments and group work prepares students for future work based dynamics and fosters skills such as leadership and co-operative learning. Despite the emphasis placed on team work in academic settings, there is still very much a place for individual work. In this study, we measured the student's own thoughts on working on their own against working in a team, a team consisting of someone they preferred or an assigned team member. The students were asked how the three working groups impacted on their enjoyment, understanding, technique and time efficiency in three sets of laboratory classes in a single academic year.

Students were asked to grade their enjoyment, understanding of the laboratory activities, their technique and how efficiently they performed (time taken to complete experiment) the three laboratory modules on a scale of 1 to 5 , with 5 indicating the highest score.

Prior to these questions the students were asked which group setting they preferred; working alone, with a preferred partner or which an assigned partner. The majority of students indicated that they preferred to work on their own against any other group work $(76 \%$, Figure 2$)$. The figure was almost as high when students who experienced all three working groups were analyzed with $71 \%$ indicating they preferred working alone against $14 \%$ who indicated a preference for working in either their preferred or an assigned partner (Figure 3). This result is not surprising, as previous studies have shown a preference for some students to working alone (Gottschall \& García-Bayotms, 2008; McManus \& Gettinger, 1996). These studies and others indicate that when 
it comes to group work, students don't feel they have complete control of the project in question as differing opinions and approaches exist within groups. Individual work affords the student sole responsibility and direction and they can manage the work at their pace (albeit within a class timeframe in this situation), they are masters of their own destiny regarding performance and academic marks for the exercise (Levin, 2005; Watkins, 2004). At times, group dynamics and perceived or actual hierarchies within groups can be detrimental to the success of a group (Anderson \& Kennedy, 2012). Figures 2 and 3 demonstrate that given the choice, students preferred individual work.

The results show that $28 \%$ of students prefer group work rather than working on their own. Team work encourages social interaction and participants can benefit from collective participation and peer learning, which might be more suitable to some student types (Springer, 1999). However, team work can also afford students some protection and can shield some students from a lack of understanding, an ability to perform particular tasks or indeed allow for 'free-riding' whereby some group members contribute or participate less than others to the exercise. However, as Hall and Buzwell (2012) indicate, the reasons for 'free-riding' can be less than clear and more complex than students simply attempting to free ride.

Despite this, Figure 4 indicates the that a clear majority of students (78.3\%) indicate a wish to have more laboratory classes run whereby they worked on their own against only $8.7 \%$ indicating they would not, $13 \%$ had no preference. This illustrates that most students recognize some innate benefits to working on their own and would do so again. Further studies should attempt to capture the specific benefits the students see in working on their own against in a team, particularly in this laboratory based setting.

The students were next asked to rate their enjoyment, understanding, technique and time efficiency when working on their own versus working with their preferred partner or an assigned partner. The students were asked to rate their enjoyment on a scale from 1 to 5 in each of the three scenarios. The concept being tested here is whether students enjoy working on their own more rather than the camaraderie and peer learning they get from working within a group, be it with their preferred partner or with an assigned partner. Despite the pressures which working on their own brings (responsibility, time management, clear understanding, ability to perform), Table 1 indicates that there was no difference in the students' enjoyment of the work regardless of which type of group they worked in. So, while Figure 2 and 3 indicate a majority prefer to work on their own, there is no less enjoyment of the work when students are placed in a group. Given that one might suggests that a happy student is a productive student, this data suggests that the students are unaffected in this regard by working under any of these working group scenarios.

When asked to rate which work setting benefitted their understanding more, differences emerged. ANOVA analysis in Table 2a shows a difference between the treatment groups and pairwise analysis indicates that the students felt their understanding was significantly improved when they worked either on their own or with their preferred partner as opposed to working with an assigned partner. This probably harks back to elements of negativity associated with group work, particularly in groups where some individuals dominate discussion and proceeding, leaving other group members dissatisfied, alienated and fearful of speaking their mind and contributing (Burke, 2011). In our study, students working on their own felt they better understood the content versus when they worked with an assigned partner, someone they may not be comfortable discussing and questioning the problem at hand, which may ultimately hinder their understanding. While working alone imparts added responsibility for their learning and understanding, it may ultimately improve their understanding as they cannot hide behind any member of a group and there cannot be any so-called groupthink. The finding that the students feels their understanding is improved when working with a preferred rather than an assigned partner likely relates to the findings by Ennen et al. (2015) who suggest that perceived trust in your team members is vital for group work success. Trust is likely to be higher in a self-formed group rather than an assigned group.

The ability to execute experimental procedures is an important learning outcome for any practical science program and our data seems to suggest that working alone improves this outcome. Data in Table 3 indicates that 
students felt there was a large disparity between their technique under the three scenarios. There was an overwhelming feeling that their technique had improved when working on their own against when they worked either with an assigned or a preferred partner. This finding should not be too surprising, though it has huge implications for learning. Students who work on their own need to take ownership of the problem at hand, and under laboratory conditions, this will at some point mean performing and executing laboratory procedures. Rather than watching and observing a partner, the student must contemplate, plan and execute on their own. All these facets contribute to good technique and it is clear the students in this study felt that working alone contributed greatly to this. No difference was observed between students working in a self-formed group versus an assigned group.

The final question related to time efficiency while working alone or within a group. Welsh et al. (2007) suggests that working in a group may slow the process down and personal observations of students suggests that group work may be a hindrance to efficient completion of experiments. It is quite common to observe students in groups double checking every aspect of the procedure with their partner before they commit to execution of a procedure. While this process has some innate advantages, it tends to slow down the process. On the flip side, one might argue that working alone may turn out to be less efficient as there is no sharing of the work and this will take a student working on their own longer to complete, this would largely depend on the complexity of the procedure and the number of concurrent steps. The results from the questionnaire (Table 4) suggests that there is no difference in the time taken to perform the experiments regardless of which work scenario. This is a beneficial finding should a teacher wish to run classes with students working on their own (to improve their technique for example). It suggests that it should not take up any more time to run these classes, despite the individual workload.

The purpose of this study was to determine the students' perspective on working alone, with their preferred partner or with an assigned partner. The results indicate that the students feel that their understanding improves when they work either on their own or with a preferred partner. The data also indicates that students' laboratory technique may be significantly improved should they perform an experiment while working alone. This is an important finding from a learning outcomes perspective. To suggest that all students should be permitted to work alone in all laboratory classes is impractical from a class management and cost point of view. However, should there be a critical procedure or activity which a student in a module must acquire, then the student should be taught this procedure while working alone. This would also benefit their understanding of these critical procedures.

The study was designed as an analysis of the students' perspectives. The study was not designed to specifically grade the understanding, techniques or time efficiencies of the students after each given set of laboratory experiments (although these skills were assessed as part of the overall module). For this reason, it is not possible to definitively determine whether working in each of the scenarios (alone/preferred/assigned) had an impact of the students' grade in the modules. Analysis of the final laboratory scores for students working in the different scenarios was performed and while the mean grades suggest the scores within the 'working alone' treatment group was higher, there was no significant difference between the scores in any groups (Table 5). There was no impact of students' overall ability, as assessed by GPA, on this analysis. Further studies will aim to specifically assess if the differing groups influence grades. Assessment design to measure technique and understanding of experiments performed post execution should be investigated. Also, a more quantitative approach could be taken to determining time efficiency by simply recording how long it takes students to complete laboratory classes.

\subsection{Conclusion}

The study indicates that having experienced working alone, working with a chosen team member and working with an assigned team member, students feel that working alone is beneficial to their laboratory technique and educators should consider allowing students to work alone if technique is a key learning outcome. 
The benefits of individual versus group work in a biology based laboratory setting

A student's understanding of a problem and its solution also benefit from either working alone or working in a self-formed group and that once again this should be taken into consideration when laboratory classes are designed.

\section{References}

Anderson, C., \& Kennedy, J. (2012). Micropolitics: A new model of status hierarchies in teams. In M. Neale \& E. Mannix (Eds.), Looking back, moving forward: A review of group and team-based research (pp. 49-80). Bingley, UK: Emerald Group Publishing. https://doi.org/10.1108/S1534-0856(2012)0000015006

Burke A. (2011). Group work: How to use groups effectively. The Journal of Effective Teaching, 11(2), 87-95.

Cohen, E. G., \& Lotan, R. A. (2014). Planning groupwork in stages. In Designing group work: Strategies for the heterogeneous classroom ( $3^{\text {rd }}$ ed., pp. 62-84). New York, USA: Teachers College Press.

Davies, W. M. (2009). Groupwork as a form of assessment: Common problems and recommended solutions. High Education, 58, 563-584. https://doi.org/10.1007/s10734-009-9216-y

De Grave, W. S, Boshuizen, H. P. A., \& Schmidt, H. G. (1996). Problem-based learning: Cognitive and metacognitive processes during problem analysis. Instructional Science, 24, 321-341. https://doi.org/10.1007/BF00118111

Dunne, E. J., \& Rawlins, M. (2000). Bridging the gap between industry and higher education: training academics to promote student teamwork. Innovations in Education and Training international, 37(4), 361-371. https://doi.org/10.1080/135580000750052973

Ennen, N. L., Stark, E., \& Lassiter, A. (2015). The importance of trust for satisfaction, motivation, and academic performance in student learning groups. Social Psychology of Education, 18(3), 615-633. https://doi.org/10.1007/s11218-015-9306-x

Gottschall, H., \& García-Bayotms, M. (2008). Student attitudes towards group work among undergraduates in business administration, education and mathematics. Educational Research Quarterly, 32(1), 3-29.

Hall, D., \& Buzwell, S. (2013). The problem of free-riding in group projects: Looking beyond social loafing as reason for non-contribution. Active Learning in Higher Education, 14, 7-49.

https://doi.org/10.1177/1469787412467123

Levin, P. (2005). Teamwork skills. In Teamwork issues and solutions in successful teamwork (pp. 13-17). Oxford, UK: Oxford University Press.

McCoy, B. (2013). Digital distractions in the classroom: Student classroom use of digital devices for non-class related purposes. Retrieved from http://digitalcommons.unl.edu/cgi/viewcontentcgi?article $=1070 \&$ context=journalismfacpub

McDonald, L. (2016). How to manage a group project. Tips for tackling your next group project successfully. Retrieved from http://www.inquiriesjournal.com/blog/posts/297/how-to-manage-group-projects

McManus, S. M., \& Gettinger, M. (1996). Teacher and student evaluations of cooperative learning and observed interactive behaviors. The Journal of Educational Research, 90, 13-22. https://doi.org/10.1080/00220671.1996.9944439

Millis, B. (2010). Why faculty should adopt cooperative learning approaches. In B. Millis (Ed.), Cooperative learning in higher education: Across the disciplines, across the academy (pp. 1-9). Virginia, USA: Stylus Publishing.

NFQ.ie. (2016). National framework of qualifications Ireland. Retrieved from http://www.qqi.ie/Pages/National-Framework-of-Qualifications-(NFQ).aspx

Oakley, B., Felder, R. M., Brent, R., \& Elhajj, I. (2003). Turning student groups into effective teams. Journal of Student Centered Learning, 2(1), 9-34.

Springer, L., Stanne, M. E., \& Donovan, S. S. (1999). Effects of small-group learning on undergraduates in science, mathematics, engineering and technology: A meta-analysis. Review of Educational Research, 69(1), 21-51. https://doi.org/10.3102/00346543069001021

Watkins, R. (2004). Group work and assessment: The handbook for economics lecturers. Economics Network. Retrieved from http://www.economicsnetwork.ac.uk/handbook/printable/groupwork.pdf 
Cronin, M., \& McCabe, A.

Watson, P. (2002, 28-29 November). Innovative teaching, teamwork and generic skills in the university environment. Paper presented at The Centre for Professional Development and The Centre for Flexible Learning, Macquarie University, Macquarie University, NSW, Australia.

Welsh, T. N., Higgins, L., Ray, M., \& Weeks, D. J. (2007). Seeing vs. believing: Is believing sufficient to activate the processes of response co-representation? Human Movement Science, 26(6), 853-866.

https://doi.org/10.1016/j.humov.2007.06.003 